\title{
IU - A Digital Application for the Graphical Examination of Interpersonal Attachment
}

\author{
Sebastian UNGER ${ }^{\mathrm{a}, 1}$, Cony THEIS $^{\mathrm{b}}$, Christina NIEDERMANN ${ }^{\mathrm{b}, \mathrm{c}}$ \\ and Thomas OSTERMANN ${ }^{\mathrm{c}}$ \\ ${ }^{a}$ Didactics and Educational Research in Health Science, \\ Witten/Herdecke University, Germany \\ ${ }^{\mathrm{b}}$ Fine Arts, University of Applied Sciences and Arts Ottersberg, Germany \\ ${ }^{\mathrm{c}}$ Department for Psychology and Psychotherapy, Witten/Herdecke University, Germany
}

\begin{abstract}
Human attachment describes the establishment of contact between two or more people leading to a closer interpersonal relationship. For measuring attachment, the use of nonverbal assessments tools including art and drawing tasks has been shown to be an alternative to conventional assessment approaches. The present study aims at evaluating the internal criterion validity of a new digital drawing tool for measuring interpersonal attachment. 68 participants took part in this pilot study and were separated in groups of two. After completion of a 10-item subscale of Social Orientation (SO) they were asked to sit opposite to the other and to hold eye contact during a three minute drawing period. Moving the pen to the upper section near the partner stood for thoughts about the other, while moving to the lower section closest to the subject implicated thoughts about oneself. The mean distance of the resulting time series of the two subjects were calculated, using the mean Euclidean distance, and compared with the difference in the SO values via linear regression. Taking all differences together a moderate correlation of $\mathrm{r}=0.298$ was observed, which however slightly missed the level of significance $(\mathrm{p}=0.09)$. We were able to find small evidence for the criterion validity of IU digital drawing tool. For future studies, other measures of similarity in the time series, i.e. the Manhattan Distance are discussed as an extension to foster the present results.
\end{abstract}

Keywords. Interpersonal attachment, digital tablet application, mental health, interactive screen recording, drawing analysis, social orientation.

\section{Introduction}

Human attachment describes the establishment of contact between two or more people leading to a closer interpersonal relationship which commonly takes place between family members or friends but also between colleagues or in therapeutic relationships between therapist and client [1]. For mental health in particular, it has been shown that interpersonal relationships are important for the health of humans [2] - 4]. Recent studies suggest that attachment disturbances or imbalances are known to be a risk factor for developing psychopathologies i.e. aggression [5].

${ }^{1}$ Sebastian Unger,Didactics and Educational Research in Health Science, Witten/Herdecke University, Alfred-Herrhausen-Straße 50, 58448 Witten, Germany; E-mail: sebastian.unger@uni-wh.de. 
Current approaches to measure the quality of such relations are mostly maternal and paternal antenatal attachment scales [6], or surveys that depend on individual time points, such as the Interpersonal Relationship Anxiety Questionnaire [7]. However, such types of measurement seem not always to be appropriate in measuring attachment of children [8] or persons with intellectual disabilities [9]. In such cases, the use of nonverbal assessment tools including art and drawing tasks has been shown to be an alternative to conventional assessment approaches [10 - 12].

In a recent proof of concept study, we described the implementation and use of a digital drawing application "IU" to measure interpersonal attachment [13] based on an examination of pixel coordinates over time. The name itself derives from the two states "I" (= being with oneself) and "U" (= being with the other). We were able to show that in a single case the attachment states of two closely familiar persons correlated strongly over the whole time of the measurement.

The present study extends the analysis by a first approach to evaluate the internal criterion validity of the IU in a bigger sample. Therefore, the app was equipped with an already proven questionnaire as an external validated instrument. Primary goal of the present study thus was to examine whether there are correlations between both, the external and internal measured values.

\section{Methods}

\subsection{Participants}

The test group, consisting of 68 participants ( 39 females, 27 males and 2 diverse people), was recruited from various fields. The age ranged from 18 to 63 with a mean age of $\bar{x}=$ $26.57 \pm 10.39$. The test group was randomly and consecutively divided into groups of two resulting in 14 female, 7 male and 13 mixed couples. Within the couples, the age did not differ $\left(\Delta_{\text {Age }}=0.32 \pm 5.21\right.$ years; $\left.t=0.36 ; p=0.720\right)$ and, within the three groups, there were couples in which the participants were familiar and unfamiliar to each other.

All participants had normal visual or normal corrected visual abilities, no physical or mental limitations and no negative or even anxious concerns when using a digital device. Thus, each participant was able to sign a consent form and to perform the drawing exercise. An ethical permission was not necessary for this study.

\subsection{Hard-and software}

The digital input device, on which the IU was installed, was a tablet of the type ASUS Transformer Mini T102HA (Figure 1). The user's input was through a battery-operated stylus, the ASUS Pen, supporting a pressure sensitivity of 1024. The tablet was equipped with a 4GB RAM memory, a 10.1"high definition touch screen with a resolution of $1280 \times$ 800, an Intel Cherry Trail Quad-Core Processor and a Windows 10 Home/Pro operating system. Furthermore, the tablet needed an installed version of the Microsoft Expression Encoder 4.0 and an activated Windows Media Player 12 in order to capture the movements of the stylus. 


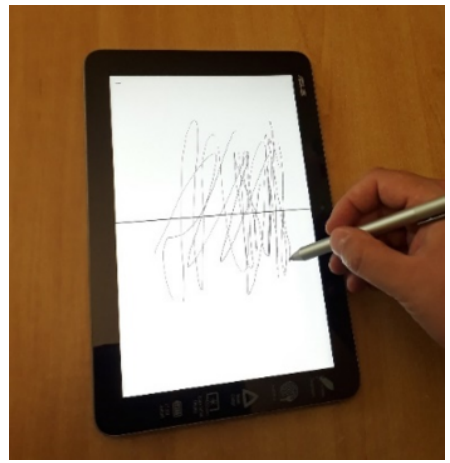

Figure 1. IU app installed on an ASUS Transformer Mini T102HA tablet

\subsection{Procedure}

First, all couples were asked to sit opposite to its study partner and, as soon as they start the drawing process, to try to hold eye contact during the whole time, even while moving the pen. Everyone received a tablet, with that any data was collected. Prior to the drawing, basic demographic data such as age and gender were asked. In addition, the 10item subscale of Social Orientation (SO) from the short version of the social skills inventory by Kanning [14] had to be answered. Because of the limitation of usual used scales for measuring attachment like the Adult Attachment Interview [15], which focus on a child-parent-relationship, this seemed as an acceptable alternative, in particular when the participants were asked to explicitly think about their opposed partner while answering the questionnaire. Thus, the purpose of this SO-scale was to serve as a marker of attachment to determine the IU's validity externally. A higher difference between the two participants therefore should correlate with a greater distance in the IU.

As next, the participants had to begin a three min (minute) long drawing exercise together with their partner. The key for a successful measurement is that they need to press the corresponding button at the same time. The start and the end were introduced with an acoustical signal. Depending on the content of their thoughts, their task was now to decide if they are connected to themselves or feel attached to their partner and to transfer this to the tablet. The interpretation of these mental states was mainly left to the participants. The interaction with the IU happened via an electronic pen. For better orientation purposes, the application presented a centerline in the middle of the screen. In the background, it was the threshold for the decision when to switch from one state to the other. The upper section near the partner ("YOU"-state) corresponded to the thoughts about the other, the lower section closest to the subject ("I"-state) to the thoughts about oneself. The starting point of both participants was the centerline. If a participant now feels bound to the other, the pen should be moved towards the direction of the partner. On the other hand, if someone is more concerned with oneself, the movement should be towards oneself. The stronger the thoughts, the further the pen should be moved away from the centerline. The pen's position should remain constant as long as the thoughts remain the same. Once the thoughts changed, the pen has to be moved to the appropriate section of the tablet, which either can be a bit closer to the centerline or completely pass it. These drawing actions were steadily repeated until the exercise was over and the participants have created a line drawing (see screenshots in Figure 2). 
At the end, the collected data was exported in XML (Extensible Markup Language) files for further processing using SPSS Version 25.

\subsection{Statistical analysis}

The movement of each participant $\mathrm{j}_{\mathrm{k}}(\mathrm{k}=1,2)$ of couple $j$ was represented as a time series $\left(x_{i}, y_{i}, t_{i}\right)_{j k}$ and transformed into two time series $f_{j k}\left(y_{i}, t_{i}\right)$. Due to the fact that attachment to the opposite was measured vertically, the horizontal position of the pen $\left(x_{i}\right)_{j k}$ was omitted for the present analysis. Thus, the mean difference between the time series was calculated, using the $\mathrm{L}_{2}$-Norm also known as Euclidian distance:

$$
d\left(f_{j 1}, f_{j 2}\right)=\left\|f_{j 1}-f_{j 2}\right\|_{2}=\sqrt{\left(f_{j 11}-f_{j 21}\right)^{2}+\cdots+\left(f_{j 1 n}-f_{j 2 n}\right)^{2}}
$$

for each couple with $j=1 \ldots n$ as suggested in [16]. The difference for each couple (also with $j=1 \ldots n$ ) between the score values of the SO-subscale was calculated as:

$$
d\left(S O_{j 1}, S O_{j 2}\right)=\left|S O_{j 1}-S O_{j 2}\right|
$$

As a measure of correlation between the distances, a linear regression together with Pearson's correlation coefficient $r$ was calculated.

\section{Results}

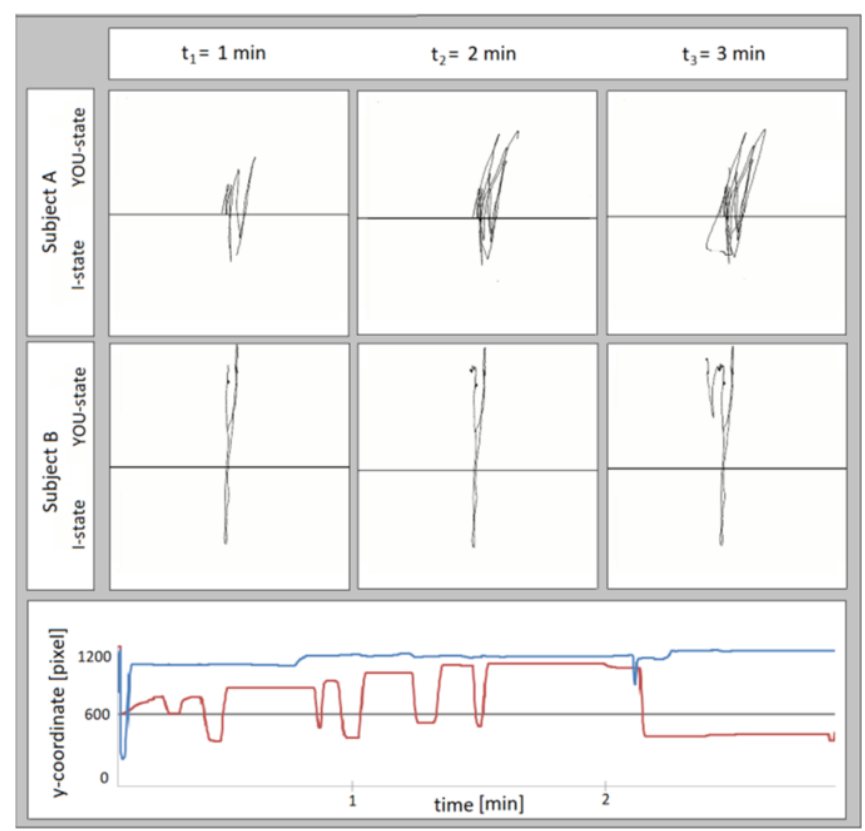

Figure 2. Example of two time series together with the respective drawings 
An example where a couple knew each other briefly is displayed in Figure 2. These two time series, whose coordinates were transferred unchanged from the XML output files, together with the respective drawings represent both types of phenomenon: areas of close attachment (i.e. between the time frame of around 90 and $120 \mathrm{~s}$ (seconds)) as well as clear distances between the two participants (i.e. from timestamp $120 \mathrm{~s}$ till the end). The differences can clearly be observed in the time curves on the bottom of the figure. Whereas subject A (red curve) tries to catch up with the other, the thoughts of subject B (blue curve) remain constant nearly during the whole process. In total, the average distance measured with the IU and the difference between the SO-subscale should, in accordance to the assumptions, be within the middle of the dataset and correlate with each other.

When considering the individual groups, only the female subgroup showed a significant correlation $(p=0.04)$ between the obtained parameters. The group of the males was far away from being significant $(p=0.34)$, as well as the mixed couples $(p=0.63)$. Despite these results, the sample couple belonging to the mixed group, which slightly knew each other, takes its expected place in the overall test group (see the red dot in the Figure 3 below).

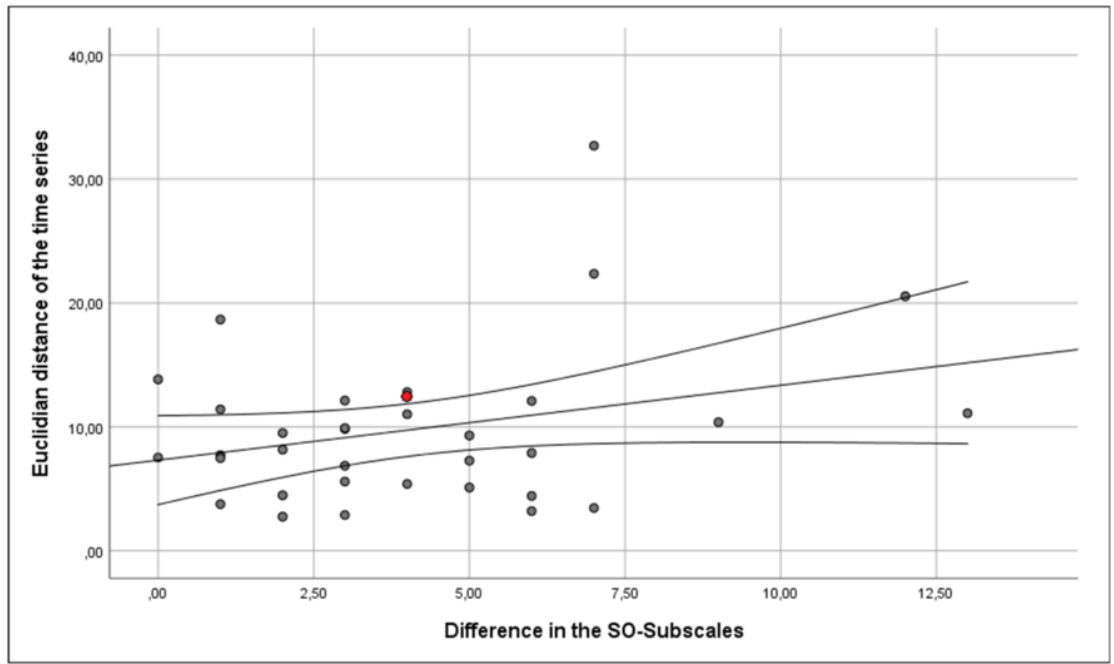

Figure 3. Regression between the observed proportionate agreements using the IU and the difference in the SO subscale.

Taking all differences of the 34 couples together, a small agreement of $r=0.298$ was observed explaining $8.9 \%$ of variance, which however slightly missed the level of significance $(p=0.09)$. The related linear regression model together with $95 \%$ confidence intervals is displayed in Figure 3. It displays a moderate linear increase $\left(\beta_{0}=7.31 ; \beta_{1}=0.6\right)$ meaning the higher SO-difference the higher the average distance value of the IU. It can also be seen, that there is a clearly large distribution within the points and that many of them are outside the confidence interval. This could be outliers that falsify the overall result or identify data that was incorrectly collected, e.g. if there was a lack of concentration during the drawing process. 


\section{Discussion}

Measuring attachment is a crucial aspect in human interaction. This article presents the results of a feasibility study to evaluate the internal criterion validity of the IU, a graphical tool to measure interpersonal attachment directly, with which better regeneration of health and more promising prevention, especially for mental health, could be achieved. Instead of approaches that measure the quality of human relations by attachment scales using a questionnaire, the IU could be an alternative for various persons with physical and mental disabilities (i.e. illiteracy or persons with cognitive impairments) without having an additional person as support.

The results of this study indicate a small but promising effectiveness of this approach by comparing the output of the app with the SO-subscale. We were able to show, that these parameters slightly correlate, which may indicate that, higher IU's average value of a couple are a diagnostic marker for their attachment to the other.

Nevertheless, our results have also a variety of limitations: Firstly, it turned out that the chosen SO-subscale measures the direct attachment to the opposite person only to a certain extent even when manipulated, especially if they were unknown to each other prior to the experiment. Therefore, further studies with the IU should recruit couples, which know each other for a longer time and should use a more reliable tool as external criterion such as the dyadic relationship scale [17]. Secondly, our approach has so far only been used for the available vertical of the drawing area. In this respect, the horizontal dimension might represent a graphical correlation of a dimensions underlying measure of adult attachment, i.e. negativity-positivity or cold-warm [18]. From the technical point of view, metrics like the Manhattan distance [19] might be a promising way to measure the distance in this two dimensional approach, as it includes the horizontal movement of the pen, which has been omitted in the present analysis.

\section{Acknowledgement}

Special thanks go to our student assistant, Marvin Beringer, who actively supported us with the collection of the dataset. Without him, our research work would not be on a presentable level.

\section{References}

[1] R. M. P. Fearon, G. I. Roisman, Attachment theory: progress and future directions, Current Opinion in Psychology 15 (2017), 131-136.

[2] A. Degnan, A. Seymour-Hyde, A. Harris and K. Berry, The role of therapist attachment in alliance and outcome: A systematic literature review, Clinical Psychology \& Psychotherapy 23 (2016), 47-65.

[3] M. J. Diener and J. M. Monroe, The relationship between adult attachment style and therapeutic alliance in individual psychotherapy: A meta-analytic review. Psychotherapy 48 (2011), 237-248.

[4] T. Ein-Dor and G. Hirschberger, Rethinking attachment theory: From a theory of relationships to a theory of individual and group survival,Current Directions in Psychological Science 25(4) (2016), 223-227

[5] R. P. Fearon, M. J. Bakermans-Kranenburg, M. H. van IJzendoorn, A. M. Lapsley and G. I. Roisman, The significance of insecure attachment and disorganization in the development of children's externalizing behavior: A meta-analytic study,Child Development 81(2) (2010), 435-456.

[6] A. Göbel, C. Barkmann, J. Goletzke, K. Hecher, M. Schulte-Markwort, P. Arck, A. Diemer and S. Mudra, Psychometric properties of 13-item versions of the maternal and paternal antenatal attachment scales in German,Journal of reproductive and infant psychology (2019), 1-13. 
[7] F. Naz and R. Kausar, Translation and Validation of Interpersonal Relationship Anxiety Questionnaire (IRAQ),FWU Journal of Social Sciences 9(1) (2015), 118-126.

[8] T. Jewell, T. Gardner, K. Susi, K. Watchorn, E. Coopey, M. Simic, P. Fonagy and I. Eisler, Attachment measures in middle childhood and adolescence: A systematic review of measurement properties, Clinical psychology review $\mathbf{6 8}$ (2019), 71-82.

[9] D. J. Gallichan and C. George, Assessing attachment status in adults with intellectual disabilities: The potential of the Adult Attachment Projective Picture System, Advances in Mental Health and Intellectual Disabilities 8(2) (2014), 103-119.

[10] S. Snir and D. Regev, ABI-Art-based intervention questionnaire, The Arts in psychotherapy 40(3) (2013), $338-346$.

[11] S. Snir and H. Wiseman, Couples' joint drawing patterns: Associations with self-report measures of interpersonal patterns and attachment styles, The Arts in Psychotherapy 48 (2016), 28-37.

[12] A. Mikhailova and D. A. Friedman, Partner Pen Play in Parallel (PPPiP): A New PPPiParadigm for Relationship Improvement, In Arts 7(3) (2018), 39.

[13] S. Unger, C. Theis, and T. Ostermann, Examination of Interpersonal Attachment with the Help of a Digital Tablet Application: A Proof of Concept Study, Proceedings of the 13th International Joint Conference on Biomedical Engineering Systems and Technologies 5 (2020), 310-315.

[14] U. P. Kanning, ISK - Inventar Sozialer Kompetenzen, Hogrefe, Göttingen, 2009.

[15] C. George, N. Kaplan and M. Main, Adult attachment Interview, unpublished manuscript, University of California, Berkeley, 1996.

[16] H. G. M. Vossen, J. T. Piotrowski and P. M. Valkenburg, Development and Validation of the Adolescent Measure of Empathy and Sympathy (AMES), Personality and Individual Differences 74 (2015), 66-71.

[17] D. Vajda, B. K. Thege and S. Rózsa, Factor Structure of the Dyadic Adjustment Scale, European Journal of Psychological Assessment. European Journal of Psychological Assessment 35 (2017), 326-334.

[18] D. W. Griffin, and K. Bartholomew, Models of the self and other: Fundamental dimensions underlying measures of adult attachment. Journal of personality and social psychology 67(3) (1994), 430-445.

[19] M. D. Malkauthekar, Analysis of euclidean distance and manhattan distance measure in face recognition, Third International Conference on Computational Intelligence and Information Technology (2013), 503507. 